\title{
Lead phytoremediation potential of sixty-one plant species: An open field survey
}

\author{
Muhammad Anwar Sajad ${ }^{1 *}$, Muhammad Saleem Khan ${ }^{1}$, Hazrat Ali ${ }^{2}$ \\ and Zaib-Un-Nisa ${ }^{1}$ \\ 1. Department of Botany, Islamia College Peshawar (A Public sector University), Khyber Pakhtunkhwa- \\ Pakistan \\ 2. Department of Zoology, University of Malakand, Chakdara, Dir Lower, Khyber Pakhtunkhwa-Pakistan \\ *Corresponding author's email: sajad.khan92@yahoo.com \\ Citation \\ Muhammad Anwar Sajad, Muhammad Saleem Khan, Hazrat Ali, Zaib-Un-Nisa. Lead phytoremediation \\ potential of sixty-one plant species: An open field survey. Pure and Applied Biology. Vol. 8, Issue 1, pp405- \\ 419. http://dx.doi.org/10.19045/bspab.2018.700200
}

\begin{tabular}{llll}
\hline \hline Received: $13 / 10 / 2018$ & Revised: 28/11/2018 & Accepted: 30/11/2018 & Online First: 08/12/2018 \\
\hline \hline
\end{tabular}

\section{Abstract}

Lead poisoning has been documented as a key public health threat, mostly in developing countries. Though several public health and occupational methods have been undertaken in order to control lead exposure, cases of lead poisoning are still reported. Exposure to lead produces various deleterious effects on the hematopoietic, renal, and reproductive and central nervous system, mainly through increased oxidative stress. These alterations play a prominent role in disease manifestations. In the current paper the concentration of lead was analysed in the soil of different sites and plant parts (roots and shoots) in their natural habitat. The concentration of lead in the soil and plant parts; roots and shoots was found in the range of 132.3 , 22-533.33 and $13.67-432 \mathrm{mg} / \mathrm{Kg}$ respectively. Bioconcentration factor (BCF), translocation factor (TF) and bioaccumulation coefficient (BAC) of all the analysed plants were calculated. The feasibility of each plant species for the phytoremediation of lead metal was evaluated on the basis of $\mathrm{BCF}, \mathrm{TF}$ and BAC. The BCFs, TFs and BACs values of the plants for lead metal was found in the range of 1.86-193.33, 0.05-8.3 and 1.79-219 respectively. Most of the plant species showed feasibility for the phytoremediation of lead metal but based on its concentration in shoots and BCFs, TFs and BACs values, Erigeron canadensis L., Stellaria media (L.) Vill. Medicago minima (L.) L., Delphinium uncinatum Hook.f. \& Thomson and Verbena officinalis L. were found the most efficient plants for the phytoextraction of lead while based on its concentration in roots and BCFs, TFs and BACs values, Nonea edgeworthii A. DC., Arabidopsis thaliana (L.) Heynh., Sarcococca saligna (D. Don) Muell.-Arg. in DC., Prodr., Marrubium vulgare L. and Ranunculus arvensis L. for the phytostabilization of lead metal.

Keywords: Lead; Phytoremediation; Phytoextraction; Phytostabilization

\section{Introduction}

One among the serious problems in the ecosphere is the contamination of the ecosystems by heavy metals. The practice of heavy metals for numerous applications has focused the release of these components into the environment. The problem of heavy metal pollution is becoming ever more severe with the industrial growth and disturbance of the usual biogeochemical cycles. Heavy metals are basically nonbiodegradable and therefore accumulate in 
the ecosystem. The accumulation of these contaminants in soil and water poses risks to the health of living organisms and ecosystems. Heavy metals accumulate in the tissues of the living organisms as they pass from the trophic levels and therefore their concentrations increase in them. In soil heavy metals cause poisonous effects on soil microorganisms, which may render their activities and numbers [1]. Heavy metals are characterized as non-essential and essential regarding their role. Essential heavy metals (iron, manganese, copper, zinc, and nickel) are needed by living organisms in small quantities [2] while nonessential heavy metals (cadmium, lead, arsenic, mercury, and chromium) are not required for vital biochemical and physiological functions by living organisms [3]. The concentration of these metals beyond the permitted limits interferes with the normal physiology of the living systems therefore pose harmful effects on health.

Lead is the most significant toxic heavy metal in the environment. Due to its main physico-chemical belongings, its use can be reviewed to historical times. Worldwide it is profusely distributed and is dangerous ecological chemical [4]. Its significant properties like malleability, softness, ductility, resistance to corrosion and poor conductibility seem to make hard to give up its use. Due to its continuous use and nonbiodegradable nature, its concentration accumulates in the environment with increasing risks. The exposure of human to lead and its compounds occurs generally in lead related occupations with numerous sources like industrial processes and leaded gasoline such as lead smelting and its combustion, pottery, lead based painting, boat building, lead containing pipes, grids, arm industry, battery recycling, printing of books, pigments etc. Still its widespread use has withdrawn in many countries of the world, it is still used in many industries like battery recycling and manufacturing, car repair, smelting, refining etc. it is a highly toxic metal affecting virtually every organ in the body. Nervous system is the frequently affected target in lead toxicity both in adults and children. In children lead toxicity is however of a greater impact than in adults. This is because their tissues internally and externally are softer than in adults. The exposure adults for longer time can lead in declined performance in some tests of cognitive performance that measure functions of the nervous system. Young children and Infants are more sensitive to even low concentrations of lead, which may contribute to learning deficits, behavioural problems and lowered IQ [5]. Exposure to lead for longer time has been documented to cause an increase in blood pressure along with anaemia, and that primarily in middle and old age people. Severe harm to the kidneys and brain, both in children and adult were found to be connected to exposure to heavy lead concentrations leading in death. High exposure to lead in pregnant women's may cause miscarriage. Long-lasting lead exposure was found to diminish fertility in males [6]. Impairment to the nervous system and blood disorders have a high incidence in lead toxicity.

A suitable in situ technique which is costeffective and environmentally sustainable for removing metals like lead from soils is represented by phytoremediation (the use of higher plants to clean up soils). One phytoremediation strategy consists of phytoextraction, employing hyper accumulator plants to concentrate metals at the shoot level $[7,8]$. Care should be taken in choosing the right hyper accumulator species for the application of phytoremediation techniques, because the introduction of alien plants may alter and disrupt indigenous ecosystems [9] and because well-known hyper accumulator species may be unsuitable for local climatic conditions [10].

In the present research work sixty one plant species belongs to thirty families were collected and analysed for the concentration of lead metal. It was analysed in the soil of the root zone, in root and shoot of each plant. Phytoremediation potential of the analysed plants grown in their natural 
habitats was evaluated by the calculation of Bioconcentration Factor (BCF), Translocation Factor (TF) and Biological Accumulation Coefficient (BAC).

\section{Materials and methods}

\section{Research area}

Lower Dir is one of the 26 districts of the Khyber Pakhtunkhwa province of Pakistan. The district was formed in 1996, when the district of Dir was divided into Upper Dir and Lower Dir. Timergara city is the district headquarters and largest city. It mainly comprises the terrain drained by the Panjkora River and its affluents. Dir takes its name from the name of a village, Dir, which served as the capital of the state during the Nawabs era Dir (princely state). It has District Swat in the East, Afghanistan on the West, Upper Dir on North-West and Malakand on the south. Pashto is the main spoken language of the population, followed by Kohistani and Gujri.

\section{Collection of plants and soil from the research area}

Sixty one plant species were collected from different locations of the research area. Soil was also collected from the root zone of each collected plant. Soil was analyzed for the background concentrations of lead. Collected plants were identified with the help of Flora of Pakistan or by matching with the already preserved specimens at the Herbarium of Islamia College University Peshawar. After identification, each plant was separated into roots and shoots. These plant parts were dried in the shade for a week and then fully dried in an oven at $75^{\circ} \mathrm{C}$ for 24 hours. Plant parts were grinded with the help of pestle and mortar. The powdered samples were digested using $\mathrm{HNO}_{3}$ and $\mathrm{HClO}_{4}$ and analyzed for the concentrations of lead using Atomic Absorption Spectroscopy (AAS). Bioconcentration factor (BCF) as well as a translocation factor (TF) of the collected plants were determined and their overall feasibility for the phytoextraction and phytostabilization was evaluated [11-13].

\section{Analysis of heavy metals in soil}

The collected soil of the root zone of each plant was analyzed for the background concentrations of lead. Heavy metals in the soil were determined according to Sharidah [14] 5g sample of the soil was taken in a 100 mL beaker. $3 \mathrm{~mL}$ of $30 \% \mathrm{H}_{2} \mathrm{O}_{2}$ was added to it. This was left undisturbed for 1 hour until the vigorous reaction ceased. Then 75 $\mathrm{mL}$ of $0.5 \mathrm{M} \mathrm{HCl}$ solution was added to it and heated on hot plate for 2 hours. The digest was filtered through a Whatman filter paper. The filtrate was used for the determination of lead by atomic absorption spectrometry. The analysis was conducted in triplicate. Results were shown as mean.

Analysis of accumulated heavy metals in plant samples

For this purpose, each plant part was thoroughly washed with tape water and then with distilled water in order to remove dusts and soil particles. The clean plant parts were dried in an oven at $105^{\circ} \mathrm{C}$ for 24 hours. Then the plant samples were grinded with the help of pestle and mortar. The powder was digested according to Awofolu [15]. $0.5 \mathrm{~g}$ sample of the plant part was taken into a $100 \mathrm{~mL}$ beaker. $5 \mathrm{~mL}$ concentrated $(65 \%) \mathrm{HNO}_{3}$ and $2 \mathrm{~mL} \mathrm{HClO}_{4}$ were added to it and heated on hot plate until the digest became clear. The digest was allowed to cool and then filtered through a Whatman filter paper. The filtrate was collected in a $50 \mathrm{~mL}$ volumetric flask and diluted to the mark with distilled water. The filtrate was used for the analysis of lead by AAS. As mentioned previously, each experiment was run in triplicate. Results were shown as mean.

\section{Evaluation of plants for the phytoextraction of lead}

Bioconcentration factor $(\mathrm{BCF})$ indicates the efficiency of a plant in up-taking heavy metals from soil and accumulating them into its tissues. "It is a ratio of the concentration of lead in the plant tissue (root, stem or leaves) to that in soil." It is calculated as follows [13].

Bioconcentration Factor $(B C F)=$ $\frac{C P b_{\text {harvested tissue }}}{C P b_{\text {soil }}} \ldots$ (1) 
where $\mathrm{C}_{\text {harvested tissue }}$ is the concentration of the metal in the plant harvested tissue (roots) and $\mathrm{C}_{\text {soil }}$ is the concentration of the same metal in soil.

Translocation factor (TF) shows the efficiency of the plant in translocating lead from roots to shoots. "It is a ratio of the concentration of lead in shoots to that in its roots." It is calculated as follows [11, 12].

Translocation Factor $(T F)=\frac{C P b_{\text {shoots }}}{C P b_{\text {roots }}} \ldots$

Whereas $C P b_{\text {shoots }}$ is the concentration of lead in shoots and $\mathrm{C} \mathrm{Pb}$ roots is the concentration of the metal in roots.

Bioaccumulation Coefficient (BAC) is the ratio of the concentration of lead in plant shoot to that in the soil and was calculated for all the studied plants according to the equation given below [16-18].

Bioaccumulation Coefficient $(B A C)=$ $\frac{C P b_{\text {shoots }}}{C P b \text { Soil }} \ldots$... (3)

Where $\mathrm{C} \mathrm{Pb}$ shoots is the concentration of lead in the shoots and $\mathrm{C} \mathrm{Pb}$ soil is the concentration of metal in soil. BCFs, TFs and BACs of the studied plants for lead were calculated according to the mentioned formulas. From these calculations, the feasibility of the plants for the phytoextraction and phytostabilization of the lead was evaluated.

\section{Statistical analysis}

Each experiment was conducted in triplicate $(n=3)$. Results are shown as mean \pm standard Deviation. Experimental data were analysed using Excel

\section{Results and discussion \\ Concentration of lead in the analysed plants}

The family, botanical name, number of site and name of the site of collection as well as the concentration of lead in the soil and plant parts (root and shoot) are shown in (Table 1). The concentration of lead in the soil of different sites and plant parts (roots and shoots) was found in the range of 1$32.3,22-533.33$ and $13.67-432 \mathrm{mg} / \mathrm{Kg}$ respectively.

Evaluation of the analysed plants for the phytoremediation of lead

Bioconcentration factor (BCF),

translocation factor (TF) and bioaccumulation coefficient (BAC) of all the analysed plants were calculated. The feasibility of each plant species for the phytoremediation; phytoextraction and phytostabilization of lead metal was evaluated. The BCFs, TFs and BACs values of the plants for lead metal was found in the range of 1.86-193.33, 0.05-8.3 and 1.79219 respectively (Table 2 ).

Concentration of lead in the soil of the analysed sites

It is clear from the data of (Table 1) that the concentration of lead in the soil of sixty one sites varies in the range of $1-32.3 \mathrm{mg} / \mathrm{Kg}$. The concentration of lead in the soil of the studied sites in $\mathrm{mg} / \mathrm{Kg}$ was found in the order: Site 30 (32.3) > Site 39 (30.5) > Site $50(28.8)>$ Site $4(28.63)>$ Site $43(25.6)>$ Site $10(24.6)>$ Site 54 (21.93) $>$ Site 12 $(21.1)>$ Site $29(19.9)>$ Site $60 \quad(18.93)>$ Site $27(18.63)>$ Site $9(17.8)>$ Site $22(17)$ $>$ Site $24(16.6)>$ Site $20(16.33)>$ Site 13 $(14.73)>$ Site $11(14.7) \geq$ Site $28(14.7)>$ Site $44(12.73)>$ Site $32(11.53)>$ Site 31 $(11.17)>$ Site $45(10.23)>$ Site $59(9.83)>$ Site $35(9.17)>$ Site $37(8.13)>$ Site 33 $(8.07)>$ Site $25(7.97)>$ Site $1(7.93)>$ Site $17(7.7)>$ Site 52 (7.6) > Site 61 (6.8) > Site $21(6.76)>$ Site $53(5.83)>$ Site $26(5.7)>$ Site $19(5.47)>$ Site $23(5.33)>$ Site 3 $(5.07)>$ Site $51(4.9)>$ Site $18(4.8)>$ Site $8(3.93)>$ Site $16(3.9)>$ Site 48 (3.27) > Site $14(2.9)>$ Site $5(2.67)>$ Site $42(2.57)$ $>$ Site $34(2.33)>$ Site $49(2.27)>$ Site 6 $(2.2)>$ Site $58(1.9)>$ Site $57(1.87)>$ Site $56(1.8)>$ Site $15(1.73)>$ Site $55(1.63)>$ Site $38(1.6)>$ Site $7(1.53) \geq$ Site 40 (1.53) $>$ Site $41(1.47)>$ Site $47(1.4)>$ Site 26 $(1.17)>$ Site $2(1.1)>$ Site 36 (1). The permissible limit of lead in soil is $32 \mathrm{mg} / \mathrm{Kg}$ [19]. Results indicate that the concentration of lead in the soil of site 30 is higher (32.3) than the allowable limit while in the rest of the sites is less than the permissible limit.

\section{Concentration of lead in the roots and} shoots of the analysed plants

Data of the (Table 1) shows that the concentration of lead was found in the roots of the plants in the range of 22-533.33 $\mathrm{mg} / \mathrm{Kg}$. Its concentration in the roots of all 
the analyzed plants in $\mathrm{mg} / \mathrm{Kg}$ was found in the order: Arabidopsis thaliana (L.) Heynh. (533.33) > Ranunculus arvensis L. (501.33) > Nonea edgeworthii A. DC. (392.66) > Sarcococca saligna (D.Don) Muell.-Arg. in DC. Prodr. (392) > Marrubium vulgare L. (309.33) > Urtica pilulifera L. (304.66) $>$ Medicago minima (L.) L. (303.33) > Calendula arvensis Boiss. (290.66) > Stellaria media (L.) Vill. (260) > Rosularia adenotricha (Wall. ex Edgew.) C.-A. Jansson (246.66) > Wulfeniopsis amherstiana (Wall. ex Benth.) D.Y. Hong (232.33) > Duchesnea indica (Jacks.) Focke (221.66) > Bryophyllum daigremontianum (Raym.-Hamet \& Perrier) A. Berger (220) $\geq$ Rosa macrophylla Lindl. (220) > Cannabis sativa L. (194) > Artemisia japonica Thunb. (188.33) > Delphinium suave Huth (177) > Xanthium strumarium L. (176) > Limonium macrorhabdon (Boiss.) O. Kuntze, Rev. Gen. (167.66) > Lactuca dissecta D.Don (129) > Iris germanica L. (127.66) > Onosma hispida Wall. ex G. Don (119.33) $>$ Plantago lanceolata L. (116.33) $\geq$ Delphinium uncinatum Hook.f. \& Thomson (116.33) > Emex spinosa (L.) Campd. (112.33) > Salvia moorcroftiana Wall. ex Benth. (112) > Filago hurdwarica (Wall. ex DC.) Wagenitz (110.66) > Silybum marianum (L.) Gaertn. (108) > Verbascum thapsus L. (101.66) > Sanguisorba minor Scop. (96.33) > Rydingia limbata (Benth.) Scheen \& V.A.Albert (91.67) > Cousinia buphthalmoides Regel (90) > Phlomoides superba (Royle ex Benth.) Kamelin \& Makhm. (88.33) > Daphne mucronata Royle (88) > Teucrium stocksianum Boiss. (86.33) > Persicaria glabra (Willd.) M. Gómez (79) > Ixiolirion tataricum (Pall.) Schult. \& Schult. f. (76) > Verbena officinalis L. (65.67) > Polygala abyssinica R.Br. ex Fresen. (64.33) > Astragalus pyrrhotrichus Boiss. (60.67) > Ajuga integrifolia Buch.-Ham. (60.53) > Isatis tinctoria L. (58.67) > Asplenium dalhousiae Hook. (58.33) > Torilis leptophylla (L.) Rchb.f. (56.67) > Sisymbrium irio L. (53) > Euphorbia helioscopia L. (50.33) > Vicia sativa L. (48) $\geq$ Geranium rotundifolium L. (48) > Himalaiella heteromalla (D.Don) RaabStraube (47.33) > Erigeron canadensis L. (45.67) > Cerastium glomeratum Thuill. (44.67) > Medicago lupulina L. (42.67) > Artemisia vulgaris L. (35) > Argyrolobium stenophyllum Boiss (32) > Pteris cretica L. (31.67) > Catharanthus roseus (L.) G. Don (31.33) > Cirsium vulgare (Savi) Ten. (30) > Cheilanthes pteridoides C. Chr. (28.67) > Allium griffithianum Boiss. (27.33) > Solanum nigrum L., Sp. Pl. (26) > Micromeria biflora (Buch.-Ham. ex D.Don) Benth. (22).The permissible limit of Lead in plants recommended by WHO is $2 \mathrm{mg} / \mathrm{Kg}$ [20]. Results showed that the concentration of Lead in the roots of all the plants is higher than that of this permissible limit.

\section{Concentration of lead in the shoots of the analysed plants}

It is evident from the data of (Table 1) that the concentration of lead in the shoots of the analyzed plant in $\mathrm{mg} / \mathrm{Kg}$ was found in the order: Arabidopsis thaliana (L.) Heynh. (432) > Medicago minima (L.) L. (394) > Erigeron canadensis L. (379) > Stellaria media (L.) Vill. (298.33) > Verbena officinalis L. (269.33) > Delphinium uncinatum Hook.f. \& Thomson (251.66) > Ranunculus arvensis L. (249) > Wulfeniopsis amherstiana (Wall. ex Benth.) D.Y. Hong (240.33) > Salvia moorcroftiana Wall. ex Benth. (239.66) > Onosma hispida Wall. ex G. Don (228.33) $>$ Marrubium vulgare L. (221.66) > Delphinium suave Huth (219.33) > Phlomoides superba (Royle ex Benth.) Kamelin \& Makhm. (219) > Cerastium glomeratum Thuill. (218.33) > Rosularia adenotricha (Wall. ex Edgew.) C.-A. Jansson (194) > Isatis tinctoria L. (189.66) $>$ Lactuca dissecta D.Don (188.66) > Ixiolirion tataricum (Pall.) Schult. \& Schult. f. (179) > Himalaiella heteromalla (D.Don) Raab-Straube (170.33)> Daphne mucronata Royle (169.33)> Bryophyllum 
daigremontianum (Raym.-Hamet \& Perrier) A. Berger (162.66) > Limonium macrorhabdon (Boiss.) O. Kuntze, Rev. Gen. (160) > Cirsium vulgare (Savi) Ten. (150.33) > Sanguisorba minor Scop. (149) $>$ Allium griffithianum Boiss. (147.33) > Sarcococca saligna (D.Don) Muell.-Arg. in DC. Prodr. (144.66) > Cannabis sativa L. (139) > Astragalus pyrrhotrichus Boiss. (138.33) > Asplenium dalhousiae Hook. (119.33) > Filago hurdwarica (Wall. ex DC.) Wagenitz (109.66) > Duchesnea indica (Jacks.) Focke (108.66) > Rydingia limbata (Benth.) Scheen \& V.A.Albert (99) $>$ Pteris cretica L. (98.67) > Euphorbia helioscopia L. (98.33) > Solanum nigrum L., Sp. Pl. (97.67) > Micromeria biflora (Buch.-Ham. ex D.Don) Benth. (97.33) > Emex spinosa (L.) Campd. (88.33) > Nonea edgeworthii A. DC. (82.33) > Vicia sativa L. (80) > Silybum marianum (L.) Gaertn. (79.67) > Plantago lanceolata L. (70.67) > Verbascum thapsus L. (69.67) > Artemisia japonica Thunb. (69.33) > Iris germanica L. (67.67) $\geq$ Rosa macrophylla Lindl. (67.67) > Medicago lupulina L. (61.67) > Catharanthus roseus (L.) G. Don (61.33) > Polygala abyssinica R.Br. ex Fresen. (56.33) > Persicaria glabra (Willd.) M. Gómez (54.33) > Xanthium strumarium L. (51.33) > Cousinia buphthalmoides Regel (50) > Silybum marianum (L.) Gaertn. (49.33) > Urtica pilulifera L. (40) > Artemisia vulgaris L. (39.67) > Argyrolobium stenophyllum Boiss (32.67) > Ajuga integrifolia Buch.-Ham. (31) > Cheilanthes pteridoides C. Chr. (28.67) > Torilis leptophylla (L.) Rchb.f. (26.33) > Teucrium stocksianum Boiss. (24.67) > Geranium rotundifolium L. (20.67) > Calendula arvensis Boiss. (13.67).The permissible limit of Lead in plants recommended by WHO is $2 \mathrm{mg} / \mathrm{Kg}$ [20]. Results showed that the concentration of Lead in the shoots of all the plants is higher than this permissible limit.

Bioconcentration factor (BCF) of the analysed plants for lead
It is clear from the data of (Table 2) that the Bioconcentration factor (BCF) was calculated as lead concentration ratio of plant roots to soil $[18,21,22]$. The calculated bioconcentration factor (BCF) of all the plants was in the order: Marrubium vulgare L. (193.33) > Calendula arvensis Boiss. (189.97) > Rosa macrophylla Lindl. (134.97) > Wulfeniopsis amherstiana (Wall. ex Benth.) D.Y. Hong (124.91) > Arabidopsis thaliana (L.) Heynh. (111.11) > Xanthium strumarium L. (101.73) > Nonea edgeworthii A. DC. (100.68) > Emex spinosa (L.) Campd. (96.01) > Phlomoides superba (Royle ex Benth.) Kamelin \& Makhm. (88.33) > Salvia moorcroftiana Wall. ex Benth. (73.2) > Artemisia japonica Thunb. (70.54) > Ranunculus arvensis L. (65.97) > Teucrium stocksianum Boiss. (58.73) > Verbascum thapsus L. (56.48) > Persicaria glabra (Willd.) M. Gómez (56.43) > Torilis leptophylla (L.) Rchb. f. (51.52) > Rosularia adenotricha (Wall. ex Edgew.) C.-A. Jansson (43.27) > Duchesnea indica (Jacks.) Focke (38.02) > Silybum marianum (L.) Gaertn. (37.24) > Cannabis sativa L. (36.4) > Delphinium suave Huth (36.12) > Ixiolirion tataricum (Pall.) Schult. \& Schult. f. (32.62) > Bryophyllum daigremontianum (Raym.Hamet \& Perrier) A. Berger (27.6) > Astragalus pyrrhotrichus Boiss. (23.61) > Sarcococca saligna (D.Don) Muell.-Arg. in DC. Prodr. (23.06) > Urtica pilulifera L. (16.09) > Stellaria media (L.) Vill. (15.92) $>$ Artemisia vulgaris L. (15.91) > Iris germanica L. (15.82) > Onosma hispida Wall. ex G. Don (15.5) > Pteris cretica L. (13.95) > Solanum nigrum L., Sp. Pl. (13.68) > Limonium macrorhabdon (Boiss.) O. Kuntze, Rev. Gen. (13.17) > Isatis tinctoria L. (10.73) > Verbena officinalis L. (9.66) > Medicago minima (L.) L. (9.39) > Daphne mucronata Royle (8.95) > Cheilanthes pteridoides C. Chr. (8.77) > Sisymbrium irio L. (7.84) > Cirsium vulgare (Savi) Ten. (7.63) > Filago hurdwarica (Wall. ex DC.) Wagenitz (7.53) > Ajuga integrifolia 
Buch.-Ham. (6.6) > Polygala abyssinica R.Br. ex Fresen. (6.29) > Catharanthus roseus (L.) G. Don (6.18) > Lactuca dissecta D.Don (6.11) > Cousinia buphthalmoides Regel (5.06) > Plantago lanceolata L. (4.54) > Sanguisorba minor Scop. (4.39) > Vicia sativa L. (4.3)> Geranium rotundifolium $\mathrm{L}$. (4.16) > Delphinium uncinatum Hook.f. \& Thomson (4.04) > Allium griffithianum Boiss. (3.45) > Himalaiella heteromalla (D.Don) Raab-Straube (3.21) > Rydingia limbata (Benth.) Scheen \& V.A.Albert (3.01) > Micromeria biflora (Buch.-Ham. ex D.Don) Benth. (2.71) > Euphorbia helioscopia L. (2.7) > Cerastium glomeratum Thuill. (2.69) > Argyrolobium stenophyllum Boiss (2.18) > Medicago lupulina L. (2.14) > Asplenium dalhousiae Hook. (2.04) > Erigeron canadensis

L. (1.86). Sheoran et al. [23] stated that the plants are not feasible for the phytoextraction of metal if bioconcentration factor is less than one. Fitz and Wenzel [24] demonstrated that plants exhibiting $\mathrm{BCF}$ value less than one are unsuitable for the phytoextraction of metals. Results showed that the calculated bioconcentration factor of all the plants was greater than one.

\section{Translocation factor (TF) of the analysed plants for lead}

Data of (Table 2) indicates that the Translocation Factor (TF) was described as ratio of lead in plant shoot to that in plant root [16-18, 21]. The translocation factor of the plants was found in the order: Erigeron canadensis L. (8.3) > Allium griffithianum Boiss. (5.39) > Cirsium vulgare (Savi) Ten. (5.01) > Cerastium glomeratum Thuill. (4.89) > Micromeria biflora (Buch.-Ham. ex D.Don) Benth. (4.42) > Verbena officinalis L. (4.1) > Solanum nigrum L., Sp. Pl. (3.76) > Himalaiella heteromalla (D.Don) Raab-Straube (3.6) > Isatis tinctoria L. (3.23) > Pteris cretica L. (3.16) > Phlomoides superba (Royle ex Benth.) Kamelin \& Makhm. (2.48) >
Ixiolirion tataricum (Pall.) Schult. \& Schult. f. (2.36) > Astragalus pyrrhotrichus Boiss. (2.28) > Delphinium uncinatum Hook.f. \& Thomson (2.16) > Salvia moorcroftiana Wall. ex Benth. (2.14) > Asplenium dalhousiae Hook. (2.05) > Catharanthus roseus (L.) G. Don (1.96) > Euphorbia helioscopia L. (1.95) > Daphne mucronata Royle (1.92)> Onosma hispida Wall. ex G. Don (1.91)> Vicia sativa L. (1.67) > Sanguisorba minor Scop. (1.55) > Sisymbrium irio L. (1.5) > Lactuca dissecta D.Don (1.46) > Medicago lupulina L. (1.45) > Medicago minima (L.) L. (1.3) > Delphinium suave Huth (1.24) > Stellaria media (L.) Vill. (1.15) > Artemisia vulgaris L. (1.13) > Rydingia limbata (Benth.) Scheen \& V.A.Albert (1.08) > Wulfeniopsis amherstiana (Wall. ex Benth.) D.Y. Hong (1.03) > Argyrolobium stenophyllum Boiss (1.02) > Filago hurdwarica (Wall. ex DC.) Wagenitz (1) $\geq$ Cheilanthes pteridoides $C$. Chr. (1) > Limonium macrorhabdon (Boiss.) O. Kuntze, Rev. Gen. (0.95) > Polygala abyssinica R.Br. ex Fresen. (0.88) > Arabidopsis thaliana (L.) Heynh. (0.81) > Rosularia adenotricha (Wall. ex Edgew.) C.-A. Jansson (0.79) $\geq$ Emex spinosa (L.) Campd. (0.79) > Bryophyllum daigremontianum (Raym.Hamet \& Perrier) A. Berger (0.74) > Cannabis sativa L. (0.72) $\geq$ Marrubium vulgare L. (0.72) > Persicaria glabra (Willd.) M. Gómez $(0.69) \geq$ Verbascum thapsus L. (0.69) > Plantago lanceolata L. (0.61) > Cousinia buphthalmoides Regel (0.56) > Iris germanica L. (0.53) > Ajuga integrifolia Buch.-Ham. (0.51) > Ranunculus arvensis L. (0.5) > Duchesnea indica (Jacks.) Focke (0.49) > Torilis leptophylla (L.) Rchb.f. (0.47) > Silybum marianum (L.) Gaertn. (0.46) > Geranium rotundifolium L. (0.43) > Artemisia japonica Thunb. (0.37) $\geq$ Sarcococca saligna (D.Don) Muell.-Arg. in DC. Prodr. (0.37) > Rosa macrophylla Lindl. (0.31) > Xanthium strumarium L. (0.29) $\geq$ Teucrium stocksianum Boiss. (0.29) > Nonea edgeworthii A. DC. (0.21) 
> Urtica pilulifera L. (0.13) > Calendula arvensis Boiss. (0.05). Translocation factor value $>$ than one indicates translocation of metal from root to above ground part [25]. Results showed that the TF value of Erigeron canadensis L. (8.3), Allium griffithianum Boiss. (5.39), Cirsium vulgare (Savi) Ten. (5.01), Cerastium glomeratum Thuill. (4.89), Micromeria biflora (Buch.-Ham. ex D.Don) Benth. (4.42), Verbena officinalis L. (4.1), Solanum nigrum L., Sp. Pl. (3.76), Himalaiella heteromalla (D.Don) RaabStraube (3.6), Isatis tinctoria L. (3.23), Pteris cretica L. (3.16), Phlomoides superba (Royle ex Benth.) Kamelin \& Makhm. (2.48), Ixiolirion tataricum (Pall.) Schult. \& Schult. f. (2.36), Astragalus pyrrhotrichus Boiss. (2.28), Delphinium uncinatum Hook.f. \& Thomson (2.16), Salvia moorcroftiana Wall. ex Benth. (2.14), Asplenium dalhousiae Hook. (2.05), Catharanthus roseus (L.) G. Don (1.96), Euphorbia helioscopia L. (1.95), Daphne mucronata Royle (1.92), Onosma hispida Wall. ex G. Don (1.91), Vicia sativa L. (1.67), Sanguisorba minor Scop. (1.55), Sisymbrium irio L. (1.5), Lactuca dissecta D.Don (1.46), Medicago lupulina L. (1.45), Medicago minima (L.) L. (1.3), Delphinium suave Huth (1.24), Stellaria media (L.) Vill. (1.15), Artemisia vulgaris L. (1.13), Rydingia limbata (Benth.) Scheen \& V.A.Albert (1.08), Wulfeniopsis amherstiana (Wall. ex Benth.) D.Y. Hong (1.03), Argyrolobium stenophyllum Boiss (1.02), Filago hurdwarica (Wall. ex DC.) Wagenitz (1) and Cheilanthes pteridoides C. Chr. (1) is greater than one.

\section{Bioaccumulation coefficient (BAC) of the analysed plants}

It is clear from that data of (Table 2) that the Bioaccumulation (BAC) was calculated as ratio of lead metal in shoots to that in soil [16-18, 21]. The calculated bioaccumulation coefficient of each plant species was found in the order: Phlomoides superba (Royle ex Benth.) Kamelin \& Makhm. (219) > Salvia moorcroftiana Wall. ex Benth. (156.64) > Marrubium vulgare L. (138.54) > Wulfeniopsis amherstiana (Wall. ex Benth.) D.Y. Hong (129.21) > Arabidopsis thaliana (L.) Heynh. (90) > Ixiolirion tataricum (Pall.) Schult. \& Schult. f. (76.82) > Emex spinosa (L.) Campd. (75.5) > Astragalus pyrrhotrichus Boiss. (53.83) > Solanum nigrum L., Sp. Pl. (51.41) > Delphinium suave Huth (44.76) > Pteris cretica L. (43.47) > Rosa macrophylla Lindl. (41.52) $>$ Verbena officinalis L. (39.61) > Persicaria glabra (Willd.) M. Gómez (38.81) > Verbascum thapsus L. (38.71) > Cirsium vulgare (Savi) Ten. (38.25) > Isatis tinctoria L. (34.67) > Rosularia adenotricha (Wall. ex Edgew.) C.-A. Jansson (34.04) > Ranunculus arvensis L. (32.76) > Xanthium strumarium L. (29.67) $>$ Onosma hispida Wall. ex G. Don (29.65) $>$ Cannabis sativa L. (26.08) > Artemisia japonica Thunb. (25.97) > Torilis leptophylla (L.) Rchb.f. (23.94) > Nonea edgeworthii A. DC. (21.11) > Bryophyllum daigremontianum (Raym.Hamet \& Perrier) A. Berger (20.41) > Duchesnea indica (Jacks.) Focke (18.64) > Allium griffithianum Boiss. (18.58) > Stellaria media (L.) Vill. (18.27) > Artemisia vulgaris L. (18.03) > Daphne mucronata Royle (17.22) > Silybum marianum (L.) Gaertn. (17.01) > Teucrium stocksianum Boiss. (16.78) > Erigeron canadensis L. (15.41) > Cerastium glomeratum Thuill. (13.15) > Limonium macrorhabdon (Boiss.) O. Kuntze, Rev. Gen. (12.57) > Medicago minima (L.) L. (12.2) > Catharanthus roseus (L.) G. Don (12.1) > Micromeria biflora (Buch.-Ham. ex D.Don) Benth. (11.97) > Sisymbrium irio L. (11.79) > Himalaiella heteromalla (D.Don) RaabStraube (11.56) > Calendula arvensis Boiss. (8.94) $\geq$ Lactuca dissecta D.Don (8.94) > Cheilanthes pteridoides C. Chr. (8.77) > Delphinium uncinatum Hook.f. \& Thomson (8.74) > Sarcococca saligna (D.Don) Muell.-Arg. in DC. Prodr. (8.51) > Iris germanica L. (8.39) > Filago hurdwarica (Wall. ex DC.) Wagenitz (7.46) $>$ Vicia sativa L. (7.16) > Sanguisorba 
minor Scop. (6.79) > Polygala abyssinica R.Br. ex Fresen. (5.51) > Euphorbia helioscopia L. (5.28) > Asplenium dalhousiae Hook. (4.17) > Ajuga integrifolia Buch.-Ham. (3.38) > Rydingia limbata (Benth.) Scheen \& V.A.Albert (3.25) > Medicago lupulina L. (3.1) > Cousinia buphthalmoides Regel (2.81) >
Plantago lanceolata L. (2.76) > Argyrolobium stenophyllum Boiss (2.22) > Urtica pilulifera L. (2.11) > Geranium rotundifolium L. (1.79). Only plant species with $\mathrm{BCF}, \mathrm{BAC}$ and $\mathrm{TF}>$ than one have the potential for the remediation process [21]. Results showed that the BAC value of all the plants is greater than one.

Table 1. Family name, plant name, site number, name of site and concentration of lead in different parts of the plant

\begin{tabular}{|c|c|c|c|c|c|c|c|}
\hline \multirow{2}{*}{$\begin{array}{l}\text { S. } \\
\text { No }\end{array}$} & \multirow[t]{2}{*}{ Family } & \multirow[t]{2}{*}{ species } & \multirow{2}{*}{$\begin{array}{l}\mathbf{S}^{*} \\
\text { No }\end{array}$} & \multirow[t]{2}{*}{ Name of Site } & \multicolumn{3}{|c|}{$\begin{array}{c}\text { Concentration of Lead } \\
(\mathrm{mg} / \mathrm{Kg})\end{array}$} \\
\hline & & & & & Soil & Root & Shoot \\
\hline 1. & Amaryllidaceae & $\begin{array}{l}\text { Allium griffithianum } \\
\text { Boiss. }\end{array}$ & 1. & Munjae & $\begin{array}{c}7.93 \\
\pm 0.15 \\
\end{array}$ & $\begin{array}{l}27.33 \\
\pm 1.53\end{array}$ & $\begin{array}{c}147.33 \pm \\
1.53\end{array}$ \\
\hline 2. & Apiaceae & $\begin{array}{l}\text { Torilis leptophylla } \\
\text { (L.) Rchb.f. }\end{array}$ & 2. & $\begin{array}{l}\text { Thorathiga } \\
\text { (Jail) }\end{array}$ & $\begin{array}{c}1.1 \\
\pm 0.1\end{array}$ & $\begin{array}{l}56.67 \\
\pm 3.06\end{array}$ & $\begin{array}{l}26.33 \\
\pm 1.53\end{array}$ \\
\hline 3. & Apocynaceae & $\begin{array}{l}\text { Catharanthus roseus } \\
\text { (L.) G. Don }\end{array}$ & 3. & $\begin{array}{l}\text { Kheima } \\
\text { (Kally) }\end{array}$ & $\begin{array}{c}5.07 \\
\pm 0.31\end{array}$ & $\begin{array}{l}31.33 \\
\pm 3.06\end{array}$ & $\begin{array}{l}61.33 \\
\pm 1.53\end{array}$ \\
\hline 4. & Aspleniaceae & $\begin{array}{c}\text { Asplenium } \\
\text { dalhousiae Hook. }\end{array}$ & 4. & $\begin{array}{l}\text { Tissu Neher } \\
\text { (Shehzadi) }\end{array}$ & $\begin{array}{l}28.63 \\
\pm 0.35 \\
\end{array}$ & $\begin{array}{l}58.33 \\
\pm 1.53 \\
\end{array}$ & $\begin{array}{c}119.33 \pm \\
2.52\end{array}$ \\
\hline \multirow{11}{*}{5.} & \multirow{11}{*}{ Asteraceae } & $\begin{array}{c}\text { Artemisia japonica } \\
\text { Thunb. }\end{array}$ & 5. & $\begin{array}{c}\text { Behari } \\
\text { (Shorgar) }\end{array}$ & $\begin{array}{r}2.67 \\
\pm 0.15 \\
\end{array}$ & $\begin{array}{l}188.33 \\
\pm 1.53 \\
\end{array}$ & $\begin{array}{l}69.33 \\
\pm 1.53 \\
\end{array}$ \\
\hline & & Artemisia vulgaris $\mathrm{L}$. & 6. & Malakabad & $\begin{array}{c}2.2 \\
\pm 0.2\end{array}$ & $\begin{array}{l}35 \\
\pm 1 \\
\end{array}$ & $\begin{array}{l}39.67 \\
\pm 2.08 \\
\end{array}$ \\
\hline & & $\begin{array}{c}\text { Calendula arvensis } \\
\text { Boiss. }\end{array}$ & 7. & $\begin{array}{l}\text { Andhera } \\
\text { (Khwar) }\end{array}$ & $\begin{array}{r}1.53 \\
\pm 0.31 \\
\end{array}$ & $\begin{array}{c}290.66 \\
\pm 1.52 \\
\end{array}$ & $\begin{array}{l}13.67 \\
\pm 1.53 \\
\end{array}$ \\
\hline & & $\begin{array}{c}\text { Cirsium vulgare } \\
\text { (Savi) Ten. }\end{array}$ & 8. & $\begin{array}{c}\text { Manzara } \\
\text { Thanga (kalle) }\end{array}$ & $\begin{array}{c}3.93 \\
\pm 0.15 \\
\end{array}$ & $\begin{array}{l}30 \\
\pm 1 \\
\end{array}$ & $\begin{array}{c}150.33 \pm \\
2.52 \\
\end{array}$ \\
\hline & & $\begin{array}{c}\text { Cousinia } \\
\text { buphthalmoides } \\
\text { Regel }\end{array}$ & 9. & $\begin{array}{l}\text { Bar Darmal } \\
\text { (Ghar) }\end{array}$ & $\begin{array}{c}17.8 \\
\pm 0.36\end{array}$ & $\begin{array}{l}90 \\
\pm 1\end{array}$ & $\begin{array}{l}50 \\
\pm 2\end{array}$ \\
\hline & & $\begin{array}{c}\text { Erigeron } \\
\text { canadensis L. }\end{array}$ & 10. & $\begin{array}{l}\text { Bar Darmal } \\
\text { (Kalle) }\end{array}$ & $\begin{array}{r}24.6 \\
\pm 0.44 \\
\end{array}$ & $\begin{array}{l}45.67 \\
\pm 1.53\end{array}$ & $\begin{array}{c}379 \\
\pm 1.73 \\
\end{array}$ \\
\hline & & $\begin{array}{c}\text { Filago hurdwarica } \\
\text { (Wall. ex DC.) } \\
\text { Wagenitz }\end{array}$ & 11. & $\begin{array}{l}\text { Malakand } \\
\text { (Khwar) }\end{array}$ & $\begin{array}{c}14.7 \\
\pm 0.36\end{array}$ & $\begin{array}{l}110.66 \\
\pm 2.31\end{array}$ & $\begin{array}{c}109.66 \pm \\
1.53\end{array}$ \\
\hline & & $\begin{array}{l}\text { Lactuca dissecta D. } \\
\text { Don }\end{array}$ & 12. & $\begin{array}{c}\text { Muslim Abad } \\
\text { (Nawa Kalle) }\end{array}$ & $\begin{array}{l}21.1 \\
\pm 0.2 \\
\end{array}$ & $\begin{array}{l}129 \\
\pm 2 \\
\end{array}$ & $\begin{array}{c}188.66 \pm \\
2.08 \\
\end{array}$ \\
\hline & & $\begin{array}{c}\text { Himalaiella } \\
\text { heteromalla }(\mathrm{D} . \text { Don) } \\
\text { Raab-Straube }\end{array}$ & 13. & $\begin{array}{l}\text { Kuz Darmal } \\
\text { (Kalle) }\end{array}$ & $\begin{array}{l}14.73 \\
\pm 0.25\end{array}$ & $\begin{array}{l}47.33 \\
\pm 2.08\end{array}$ & $\begin{array}{c}170.33 \pm \\
2.52\end{array}$ \\
\hline & & $\begin{array}{l}\text { Silybum marianum ( } \\
\text { L.) Gaertn. }\end{array}$ & 14. & $\begin{array}{l}\text { Mian Banda } \\
\text { (Kalle) }\end{array}$ & $\begin{array}{c}2.9 \\
\pm 0.2\end{array}$ & $\begin{array}{l}108 \\
\pm 2\end{array}$ & $\begin{array}{l}49.33 \\
\pm 1.53\end{array}$ \\
\hline & & $\begin{array}{c}\text { Xanthium strumarium } \\
\text { L. }\end{array}$ & 15. & $\begin{array}{c}\text { Thoratiga } \\
\text { (Kalle) }\end{array}$ & $\begin{array}{r}1.73 \\
\pm 0.21 \\
\end{array}$ & $\begin{array}{l}176 \\
\pm 2 \\
\end{array}$ & $\begin{array}{l}51.33 \\
\pm 2.51 \\
\end{array}$ \\
\hline \multirow{2}{*}{6.} & \multirow{2}{*}{ Boraginaceae } & $\begin{array}{c}\text { Nonea edgeworthii } \\
\text { A. DC. }\end{array}$ & 16. & $\begin{array}{c}\text { Manzara } \\
\text { Thanga (Ghar) }\end{array}$ & $\begin{array}{c}3.9 \\
\pm 0.1 \\
\end{array}$ & $\begin{array}{c}392.66 \\
\pm 2.31 \\
\end{array}$ & $\begin{array}{l}82.33 \\
\pm 3.06 \\
\end{array}$ \\
\hline & & $\begin{array}{l}\text { Onosma hispida } \\
\text { Wall. ex G. Don }\end{array}$ & 17. & $\begin{array}{l}\text { Majar Camp } \\
\text { (Kalle) }\end{array}$ & $\begin{array}{c}7.7 \\
\pm 0.17\end{array}$ & $\begin{array}{c}119.33 \\
\pm 1.53\end{array}$ & $\begin{array}{c}228.33 \pm \\
2.52\end{array}$ \\
\hline
\end{tabular}


Sajad et al.

\begin{tabular}{|c|c|c|c|c|c|c|c|}
\hline \multirow{4}{*}{7.} & \multirow{4}{*}{ Brassicaceae } & $\begin{array}{l}\text { Arabidopsis thaliana } \\
\text { (L.) Heynh. }\end{array}$ & 18. & $\begin{array}{l}\text { Manzara } \\
\text { Thanga } \\
\text { (Shorgar) } \\
\end{array}$ & $\begin{array}{c}4.8 \\
\pm 0.2\end{array}$ & $\begin{array}{l}533.33 \\
\pm 2.31\end{array}$ & $\begin{array}{c}432 \\
\pm 2\end{array}$ \\
\hline & & Isatis tinctoria $\mathrm{L}$. & 19. & Shengrai & $\begin{array}{c}5.47 \\
\pm 0.25\end{array}$ & $\begin{array}{l}58.67 \\
\pm 1.15\end{array}$ & $\begin{array}{c}189.66 \pm \\
1.53\end{array}$ \\
\hline & & $\begin{array}{l}\text { Stellaria media (L.) } \\
\text { Vill. }\end{array}$ & 20. & $\begin{array}{c}\text { Koz Kally } \\
\text { (Timergara) }\end{array}$ & $\begin{array}{l}16.33 \\
\pm 0.42\end{array}$ & $\begin{array}{c}260 \\
\pm 2\end{array}$ & $\begin{array}{c}298.33 \pm \\
1.53\end{array}$ \\
\hline & & Sisymbrium irio L. & 21. & Thanga (Shah) & $\begin{array}{l}6.76 \\
\pm 0.4 \\
\end{array}$ & $\begin{array}{r}53 \\
\pm 2 \\
\end{array}$ & $\begin{array}{r}79.67 \\
\pm 1.53 \\
\end{array}$ \\
\hline 8. & Buxaceae & $\begin{array}{c}\text { Sarcococca } \\
\text { saligna (D. Don) } \\
\text { Muell-Arg. in DC. } \\
\text { Prodr. }\end{array}$ & 22. & Rabat (Chena) & $\begin{array}{c}17 \\
\pm 0.27\end{array}$ & $\begin{array}{c}392 \\
\pm 2\end{array}$ & $\begin{array}{c}144.66 \pm \\
2.516\end{array}$ \\
\hline 9. & Cannabaceae & Cannabis sativa L. & 23. & $\begin{array}{c}\text { Shehzadi } \\
\text { (Banr) }\end{array}$ & $\begin{array}{c}5.33 \\
\pm 0.45 \\
\end{array}$ & $\begin{array}{c}194 \\
\pm 2 \\
\end{array}$ & $\begin{array}{c}139 \\
\pm 1 \\
\end{array}$ \\
\hline 10. & Caryophyllaceae & $\begin{array}{c}\text { Cerastium } \\
\text { glomeratum Thuill. }\end{array}$ & 24. & $\begin{array}{l}\text { Khema } \\
\text { (Shorgar) }\end{array}$ & $\begin{array}{c}16.6 \\
\pm 0.36 \\
\end{array}$ & $\begin{array}{l}44.67 \\
\pm 1.15 \\
\end{array}$ & $\begin{array}{c}218.33 \pm \\
1.53 \\
\end{array}$ \\
\hline \multirow{2}{*}{11.} & \multirow{2}{*}{ Crassulaceae } & $\begin{array}{c}\text { Bryophyllum } \\
\text { daigremontianum } \\
\text { (Raym.-Hamet \& } \\
\text { Perrier) A. Berger }\end{array}$ & 25. & $\begin{array}{l}\text { Andhera } \\
\text { (College) }\end{array}$ & $\begin{array}{l}7.97 \\
\pm 0.5\end{array}$ & $\begin{array}{c}220 \\
\pm 2\end{array}$ & $\begin{array}{c}162.66 \pm \\
2.08\end{array}$ \\
\hline & & $\begin{array}{c}\text { Rosularia } \\
\text { adenotricha (Wall. ex } \\
\text { Edgew.) C.-A. } \\
\text { Jansson } \\
\end{array}$ & 26. & $\begin{array}{l}\text { Bar Darmal } \\
\text { (Khwar) }\end{array}$ & $\begin{array}{c}5.7 \\
\pm 0.27\end{array}$ & $\begin{array}{l}246.66 \\
\pm 1.53\end{array}$ & $\begin{array}{c}194 \\
\pm 2\end{array}$ \\
\hline 12. & Euphorbiaceae & $\begin{array}{c}\text { Euphorbia } \\
\text { helioscopia L. }\end{array}$ & 27. & $\begin{array}{l}\text { Udigram } \\
\text { (Kalle) }\end{array}$ & $\begin{array}{l}18.63 \\
\pm 0.35\end{array}$ & $\begin{array}{l}50.33 \\
\pm 1.53\end{array}$ & $\begin{array}{l}98.33 \\
\pm 1.53\end{array}$ \\
\hline \multirow{4}{*}{13.} & \multirow{4}{*}{ Fabaceae } & $\begin{array}{l}\text { Argyrolobium } \\
\text { stenophyllum } \\
\text { Boiss. }\end{array}$ & 28. & $\begin{array}{l}\text { Asharigat } \\
\text { (Pull) }\end{array}$ & $\begin{array}{c}14.7 \\
\pm 0.36\end{array}$ & $\begin{array}{l}32 \\
\pm 2\end{array}$ & $\begin{array}{l}32.67 \\
\pm 1.53\end{array}$ \\
\hline & & Medicago lupulina $\mathrm{L}$. & 29. & $\begin{array}{l}\text { Kuz Darmal } \\
\text { (Gharr) }\end{array}$ & $\begin{array}{l}19.9 \\
\pm 0.2\end{array}$ & $\begin{array}{l}42.67 \\
\pm 1.15\end{array}$ & $\begin{array}{l}61.67 \\
\pm 2.12 \\
\end{array}$ \\
\hline & & Medicago minima $\mathrm{L}$. & 30. & $\begin{array}{l}\text { Tissu Neher } \\
\text { (Ghar) }\end{array}$ & $\begin{array}{c}32.3 \\
\pm 0.36 \\
\end{array}$ & $\begin{array}{l}303.33 \\
\pm 2.89 \\
\end{array}$ & $\begin{array}{c}394 \\
\pm 2 \\
\end{array}$ \\
\hline & & Vicia sativa $\mathrm{L}$. & 31. & $\begin{array}{l}\text { Kuz Darmal } \\
\text { (Khwar) }\end{array}$ & $\begin{array}{l}11.17 \\
\pm 0.25 \\
\end{array}$ & $\begin{array}{l}48 \\
\pm 2 \\
\end{array}$ & $\begin{array}{l}80 \\
\pm 2 \\
\end{array}$ \\
\hline 14. & Geraniaceae & $\begin{array}{c}\text { Geranium } \\
\text { rotundifolium L. }\end{array}$ & 32. & $\begin{array}{l}\text { Mian Banda } \\
\text { (Shorgar) }\end{array}$ & $\begin{array}{l}11.53 \\
\pm 0.31 \\
\end{array}$ & $\begin{array}{l}48 \\
\pm 2\end{array}$ & $\begin{array}{l}20.67 \\
\pm 2.52 \\
\end{array}$ \\
\hline 15. & Iridaceae & Iris germanica $\mathrm{L}$. & 33. & $\begin{array}{l}\text { Tissue Neher } \\
(\text { Kalle })\end{array}$ & $\begin{array}{c}8.07 \\
\pm 0.25 \\
\end{array}$ & $\begin{array}{l}127.66 \\
\pm 1.53 \\
\end{array}$ & $\begin{array}{l}67.67 \\
\pm 1.53 \\
\end{array}$ \\
\hline 16. & Ixioliriaceae & $\begin{array}{c}\text { Ixiolirion tataricum } \\
\text { (Pall.) Schult. \& } \\
\text { Schult. F. }\end{array}$ & 34. & Khall (Ghar) & $\begin{array}{c}2.33 \\
\pm 0.38\end{array}$ & $\begin{array}{l}76 \\
\pm 1\end{array}$ & $\begin{array}{l}179 \\
\pm 2\end{array}$ \\
\hline \multirow{4}{*}{17.} & \multirow{4}{*}{ Lamiaceae } & $\begin{array}{c}\text { Ajuga integrifolia } \\
\text { Buch.-Ham. }\end{array}$ & 35. & $\begin{array}{l}\text { Jabagai (Osai } \\
\text { Ghar) }\end{array}$ & $\begin{array}{c}9.17 \\
\pm 0.25 \\
\end{array}$ & $\begin{array}{l}60.53 \\
\pm 0.64 \\
\end{array}$ & $\begin{array}{l}31 \\
\pm 2 \\
\end{array}$ \\
\hline & & $\begin{array}{l}\text { Phlomoides superba } \\
\text { (Royle ex Benth.) } \\
\text { Kamelin \& Makhm. }\end{array}$ & 36. & $\begin{array}{l}\text { Nara Thangai } \\
\text { (Ghar) }\end{array}$ & $\begin{array}{c}1 \\
\pm 0.17\end{array}$ & $\begin{array}{l}88.33 \\
\pm 1.53\end{array}$ & $\begin{array}{c}219 \\
\pm 2\end{array}$ \\
\hline & & $\begin{array}{l}\text { Micromeria biflora } \\
\text { (Buch.-Ham. ex } \\
\text { D.Don) Benth. }\end{array}$ & 37. & Ranai & $\begin{array}{l}8.13 \\
\pm 0.2\end{array}$ & $\begin{array}{l}22 \\
\pm 3\end{array}$ & $\begin{array}{l}97.33 \\
\pm 1.53 \\
\end{array}$ \\
\hline & & $\begin{array}{l}\text { Marrubium vulgare } \\
\text { L. }\end{array}$ & 38. & $\begin{array}{c}\text { Khadang Ghar } \\
\text { (Bar Darmal) }\end{array}$ & $\begin{array}{c}1.6 \\
\pm 0.26\end{array}$ & $\begin{array}{l}309.33 \\
\pm 1.53\end{array}$ & $\begin{array}{c}221.66 \pm \\
3.51\end{array}$ \\
\hline
\end{tabular}




\begin{tabular}{|c|c|c|c|c|c|c|c|}
\hline & & $\begin{array}{c}\text { Rydingia limbata } \\
\text { (Benth.) Scheen \& } \\
\text { V.A. Albert }\end{array}$ & 39. & Behari (Ghar) & $\begin{array}{c}30.5 \\
\pm 0.44\end{array}$ & $\begin{array}{l}91.67 \\
\pm 1.53\end{array}$ & $\begin{array}{l}99 \\
\pm 1\end{array}$ \\
\hline & & $\begin{array}{l}\text { Salvia moorcroftiana } \\
\text { Wall. ex Benth. }\end{array}$ & 40. & $\begin{array}{l}\text { Majar Camp } \\
\text { (Khwar) }\end{array}$ & $\begin{array}{c}1.53 \\
\pm 0.25\end{array}$ & $\begin{array}{c}112 \\
\pm 2.52\end{array}$ & $\begin{array}{c}239.66 \pm \\
2.81\end{array}$ \\
\hline & & $\begin{array}{c}\text { Teucrium } \\
\text { stocksianum Boiss. }\end{array}$ & 41. & Sado & $\begin{array}{c}1.47 \\
\pm 0.15 \\
\end{array}$ & $\begin{array}{l}86.33 \\
\pm 1.52 \\
\end{array}$ & $\begin{array}{l}24.67 \\
\pm 1.53 \\
\end{array}$ \\
\hline 18. & Papilionaceae & $\begin{array}{c}\text { Astragalus } \\
\text { pyrrhotrichus Boiss. }\end{array}$ & 42. & Beghamdara & $\begin{array}{c}2.57 \\
\pm 0.31 \\
\end{array}$ & $\begin{array}{l}60.67 \\
\pm 1.53 \\
\end{array}$ & $\begin{array}{c}138.33 \pm \\
1.53\end{array}$ \\
\hline 19. & Plantaginaceae & $\begin{array}{l}\text { Plantago lanceolata } \\
\text { L. }\end{array}$ & 43. & Behari (Kalle) & $\begin{array}{l}25.6 \\
\pm 0.1\end{array}$ & $\begin{array}{l}116.33 \\
\pm 1.53\end{array}$ & $\begin{array}{l}70.67 \\
\pm 1.53\end{array}$ \\
\hline 20. & Plumbaginaceae & $\begin{array}{c}\text { Limonium } \\
\text { macrorhabdon } \\
\text { Boiss.) O. Kuntze, } \\
\text { Rev. Gen. }\end{array}$ & 44. & $\begin{array}{l}\text { Jabagai (Thor } \\
\text { Baba) }\end{array}$ & $\begin{array}{l}12.73 \\
\pm 0.32\end{array}$ & $\begin{array}{c}167.66 \\
\pm 1.53\end{array}$ & $\begin{array}{c}160 \\
\pm 2.65\end{array}$ \\
\hline 21. & Polygalaceae & $\begin{array}{l}\text { Polygala abyssinica } \\
\text { R.Br. ex Fresen. }\end{array}$ & 45. & $\begin{array}{l}\text { Shehzadi } \\
\text { (Kundao) }\end{array}$ & $\begin{array}{l}10.23 \\
\pm 0.32 \\
\end{array}$ & $\begin{array}{r}64.33 \\
\pm 2.08 \\
\end{array}$ & $\begin{array}{l}56.33 \\
\pm 1.53 \\
\end{array}$ \\
\hline 22 & Polvo & $\begin{array}{c}\text { Emex spinosa (L.) } \\
\text { Campd. }\end{array}$ & 46. & Haji Abad & $\begin{array}{c}1.17 \\
\pm 0.15\end{array}$ & $\begin{array}{c}112.33 \\
\pm 1.53 \\
\end{array}$ & $\begin{array}{l}88.33 \\
\pm 1.53 \\
\end{array}$ \\
\hline 22. & Polye & $\begin{array}{l}\text { Persicaria glabra } \\
\text { (Willd.) M. Gómez }\end{array}$ & 47. & $\begin{array}{l}\text { Udigram } \\
\text { (Ghar) }\end{array}$ & $\begin{array}{c}1.4 \\
\pm 0.2 \\
\end{array}$ & $\begin{array}{r}79 \\
\pm 1 \\
\end{array}$ & $\begin{array}{l}54.33 \\
\pm 1.53 \\
\end{array}$ \\
\hline & & $\begin{array}{c}\text { Cheilanthes } \\
\text { pteridoides C. Chr. }\end{array}$ & 48. & Haya Serai & $\begin{array}{c}3.27 \\
\pm 0.25\end{array}$ & $\begin{array}{l}28.67 \\
\pm 1.53 \\
\end{array}$ & $\begin{array}{l}28.67 \\
\pm 1.53 \\
\end{array}$ \\
\hline 23. & Pteridaceae & Pteris cretica L. & 49. & $\begin{array}{l}\text { Behari } \\
\text { (Khwar) }\end{array}$ & $\begin{array}{c}2.27 \\
\pm 0.23\end{array}$ & $\begin{array}{r}31.67 \\
\pm 1.527\end{array}$ & $\begin{array}{c}98.67 \\
\pm 1.527\end{array}$ \\
\hline & & $\begin{array}{c}\text { Delphinium } \\
\text { uncinatum Hook.f. \& } \\
\text { Thomson }\end{array}$ & 50. & $\begin{array}{c}\text { Bar Darmal } \\
\text { (Shorgur) }\end{array}$ & $\begin{array}{l}28.8 \\
\pm 0.2\end{array}$ & $\begin{array}{l}116.33 \\
\pm 1.527\end{array}$ & $\begin{array}{c}251.66 \pm \\
1.527\end{array}$ \\
\hline 24. & Ranunculaceae & $\begin{array}{l}\text { Delphinium suave } \\
\text { Huth }\end{array}$ & 51. & Lajbok & $\begin{array}{c}4.9 \\
\pm 0.2\end{array}$ & $\begin{array}{l}177 \\
\pm 2\end{array}$ & $\begin{array}{c}219.33 \pm \\
2.52\end{array}$ \\
\hline & & $\begin{array}{c}\text { Ranunculus arvensis } \\
\text { L. }\end{array}$ & 52. & Murnera & $\begin{array}{c}7.6 \\
\pm 0.2\end{array}$ & $\begin{array}{l}501.33 \\
\pm 1.154 \\
\end{array}$ & $\begin{array}{c}249 \\
\pm 3.605\end{array}$ \\
\hline & & $\begin{array}{l}\text { Duchesnea indica } \\
\text { (Jacks.) Focke }\end{array}$ & 53. & $\begin{array}{c}\text { Bar Darmal } \\
\text { (School) }\end{array}$ & $\begin{array}{c}5.83 \\
\pm 0.21 \\
\end{array}$ & $\begin{array}{c}221.66 \\
\pm 3.06 \\
\end{array}$ & $\begin{array}{c}108.66 \pm \\
2.52 \\
\end{array}$ \\
\hline 25. & Rosaceae & $\begin{array}{l}\text { Sanguisorba minor } \\
\text { Scop. }\end{array}$ & 54. & Rabat (Kalle) & $\begin{array}{l}21.93 \\
\pm 0.31 \\
\end{array}$ & $\begin{array}{r}96.33 \\
\pm 1.53 \\
\end{array}$ & $\begin{array}{c}149 \\
\pm 2 \\
\end{array}$ \\
\hline & & $\begin{array}{c}\text { Rosa macrophylla } \\
\text { Lindl. }\end{array}$ & 55. & $\begin{array}{l}\text { Shezadi } \\
\text { (Konahi) }\end{array}$ & $\begin{array}{c}1.63 \\
\pm 0.15\end{array}$ & $\begin{array}{c}220 \\
\pm 2\end{array}$ & $\begin{array}{l}67.67 \\
\pm 2.52 \\
\end{array}$ \\
\hline & & $\begin{array}{c}\text { Verbascum thapsus } \\
\text { L. }\end{array}$ & 56. & Khongai & $\begin{array}{c}1.8 \\
\pm 0.2 \\
\end{array}$ & $\begin{array}{l}101.66 \\
\pm 2.52 \\
\end{array}$ & $\begin{array}{l}69.67 \\
\pm 2.08 \\
\end{array}$ \\
\hline 26. & Scrophulariaceae & $\begin{array}{c}\text { Wulfeniopsis } \\
\text { amherstiana (Wall. } \\
\text { Ex Benth.) D.Y. } \\
\text { Hong }\end{array}$ & 57. & $\begin{array}{l}\text { Nara Thangai } \\
\text { (Kalle) }\end{array}$ & $\begin{array}{c}1.87 \\
\pm 0.12\end{array}$ & $\begin{array}{c}232.33 \\
\pm 2.08\end{array}$ & $\begin{array}{c}240.33 \pm \\
1.53\end{array}$ \\
\hline 27. & Solanaceae & $\begin{array}{l}\text { Solanum nigrum L., } \\
\text { Sp. Pl. }\end{array}$ & 58. & $\begin{array}{l}\text { Nawe Kalle } \\
\text { (Pori Ghar) }\end{array}$ & $\begin{array}{c}1.9 \\
\pm 0.3\end{array}$ & $\begin{array}{l}26 \\
\pm 2 \\
\end{array}$ & $\begin{array}{l}97.67 \\
\pm 1.53\end{array}$ \\
\hline 28. & Thymelaeaceae & $\begin{array}{c}\text { Daphne } \\
\text { mucronata Royle }\end{array}$ & 59. & $\begin{array}{l}\text { Balambat } \\
\text { (Chaoni) }\end{array}$ & $\begin{array}{c}9.83 \\
\pm 0.21\end{array}$ & $\begin{array}{l}88 \\
\pm 2\end{array}$ & $\begin{array}{c}169.33 \pm \\
1.53\end{array}$ \\
\hline 29. & Urticaceae & Urtica pilulifera $\mathrm{L}$. & 60. & Timur & $\begin{array}{l}18.93 \\
\pm 0.25\end{array}$ & $\begin{array}{c}304.66 \\
\pm 1.15\end{array}$ & $\begin{array}{l}40 \\
\pm 2\end{array}$ \\
\hline 30. & Verbenaceae & Verbena officinalis $\mathrm{L}$. & 61. & $\begin{array}{c}\text { Thraskun } \\
\text { Hotel } \\
\text { (Timergara) } \\
\end{array}$ & $\begin{array}{c}6.8 \\
\pm 0.2\end{array}$ & $\begin{array}{l}65.67 \\
\pm 1.53\end{array}$ & $\begin{array}{c}269.33 \pm \\
1.53\end{array}$ \\
\hline
\end{tabular}

$\mathrm{S} *$ No $=$ Site number, Concentration of lead in soil and plant parts is shown as mean $(n=3) \pm S D$ 
Table 2. Name of the plant Species and their Bioconcentration Factor, Translocation Factor and Bioaccumulation Coefficient for Lead

\begin{tabular}{|c|c|c|c|c|c|}
\hline \multirow[t]{2}{*}{ S. No } & \multirow[t]{2}{*}{ Plant Species } & \multicolumn{3}{|c|}{$\begin{array}{c}\text { Bioconcentration Factor, } \\
\text { Translocation Factor and } \\
\text { Bioaccumulation } \\
\text { Coefficient } \\
\end{array}$} & \multirow[t]{2}{*}{$\begin{array}{l}\text { Feasibility of the } \\
\text { plant for the } \\
\text { phytoremediation } \\
\text { of Lead }\end{array}$} \\
\hline & & BCF & TF & BAC & \\
\hline 1. & Allium griffithianum Boiss. & 3.45 & 5.39 & 18.58 & $++^{* *}$ \\
\hline 2. & Torilis leptophylla (L.) Rchb.f. & 51.52 & 0.47 & 23.94 & $+*$ \\
\hline 3. & Catharanthus roseus (L.) G. Don & 6.18 & 1.96 & 12.1 & $++^{* *}$ \\
\hline 4. & Asplenium dalhousiae Hook. & 2.04 & 2.05 & 4.17 & $++^{* * *}$ \\
\hline 5. & Artemisia japonica Thunb. & 70.54 & 0.37 & 25.97 & $+*$ \\
\hline 6. & Artemisia vulgaris $\mathrm{L}$. & 15.91 & 1.13 & 18.03 & $++* *$ \\
\hline 7. & Calendula arvensis Boiss. & 189.97 & 0.05 & 8.94 & $+*$ \\
\hline 8. & Cirsium vulgare (Savi) Ten. & 7.63 & 5.01 & 38.25 & $++^{* * *}$ \\
\hline 9. & Cousinia buphthalmoides Regel & 5.06 & 0.56 & 2.81 & $+*$ \\
\hline 10. & Erigeron canadensis L. & 1.86 & 8.3 & 15.41 & $++* *$ \\
\hline 11. & $\begin{array}{c}\text { Filago hurdwarica (Wall. ex DC.) } \\
\text { Wagenitz }\end{array}$ & 7.53 & 1 & 7.46 & $++* *$ \\
\hline 12. & Lactuca dissecta D. Don & 6.11 & 1.46 & 8.94 & $++* *$ \\
\hline 13. & $\begin{array}{l}\text { Himalaiella heteromalla (D. Don) } \\
\text { Raab-Straube }\end{array}$ & 3.21 & 3.6 & 11.56 & $++* *$ \\
\hline 14. & Silybum marianum (L.) Gaertn. & 37.24 & 0.46 & 17.01 & $+*$ \\
\hline 15. & Xanthium strumarium L. & 101.73 & 0.29 & 29.67 & $+*$ \\
\hline 16. & Nonea edgeworthii A. DC. & 100.68 & 0.21 & 21.11 & $+*$ \\
\hline 17. & Onosma hispida Wall. ex G. Don & 15.5 & 1.91 & 29.65 & $++^{* * *}$ \\
\hline 18. & Arabidopsis thaliana (L.) Heynh. & 111.11 & 0.81 & 90 & $+^{*}$ \\
\hline 19. & Isatis tinctoria $\mathrm{L}$. & 10.73 & 3.23 & 34.67 & $++* *$ \\
\hline 20. & Stellaria media (L.) Vill. & 15.92 & 1.15 & 18.27 & $++* *$ \\
\hline 21. & Sisymbrium irio L. & 7.84 & 1.5 & 11.79 & $++* *$ \\
\hline 22. & $\begin{array}{l}\text { Sarcococca saligna (D. Don) } \\
\text { Muell.-Arg. in DC., Prodr. }\end{array}$ & 23.06 & 0.37 & 8.51 & $+^{*}$ \\
\hline 23. & Cannabis sativa $\mathrm{L}$. & 36.4 & 0.72 & 26.08 & $+*$ \\
\hline 24. & Cerastium glomeratum Thuill. & 2.69 & 4.89 & 13.15 & $++^{* * *}$ \\
\hline 25. & $\begin{array}{c}\text { Bryophyllum daigremontianum } \\
\text { (Raym.-Hamet \& Perrier) A. } \\
\text { Berger }\end{array}$ & 27.6 & 0.74 & 20.41 & $+^{*}$ \\
\hline 26. & $\begin{array}{l}\text { Rosularia adenotricha (Wall. ex } \\
\text { Edgew.) C.-A. Jansson }\end{array}$ & 43.27 & 0.79 & 34.04 & $+*$ \\
\hline 27. & Euphorbia helioscopia L. & 2.7 & 1.95 & 5.28 & $++* *$ \\
\hline 28. & Argyrolobium stenophyllum Boiss. & 2.18 & 1.02 & 2.22 & $++* *$ \\
\hline 29. & Medicago lupulina $\mathrm{L}$. & 2.14 & 1.45 & 3.1 & $++^{* *}$ \\
\hline 30. & Medicago minima (L.) L. & 9.39 & 1.3 & 12.2 & $++^{* *}$ \\
\hline 31. & Vicia sativa $\mathrm{L}$. & 4.3 & 1.67 & 7.16 & $++^{* *}$ \\
\hline 32. & Geranium rotundifolium $\mathrm{L}$. & 4.16 & 0.43 & 1.79 & $+*$ \\
\hline 33. & Iris germanica $\mathrm{L}$. & 15.82 & 0.53 & 8.39 & $+*$ \\
\hline 34. & $\begin{array}{l}\text { Ixiolirion tataricum (Pall.) Schult. } \\
\qquad \text { Schult. f. }\end{array}$ & 32.62 & 2.36 & 76.82 & $++^{* *}$ \\
\hline 35. & Ajuga integrifolia Buch.-Ham. & 6.6 & 0.51 & 3.38 & $+*$ \\
\hline 36. & $\begin{array}{l}\text { Phlomoides superba (Royle ex } \\
\text { Benth.) Kamelin \& Makhm. }\end{array}$ & 88.33 & 2.48 & 219 & $++* *$ \\
\hline 37. & $\begin{array}{l}\text { Micromeria biflora (Buch.-Ham. ex } \\
\text { D.Don) Benth. }\end{array}$ & 2.71 & 4.42 & 11.97 & $++* *$ \\
\hline
\end{tabular}




\begin{tabular}{|c|c|c|c|c|c|}
\hline 38. & Marrubium vulgare L. & 193.33 & 0.72 & 138.54 & $+*$ \\
\hline 39. & $\begin{array}{c}\text { Rydingia limbata (Benth.) Scheen } \\
\text { \& V.A. Albert }\end{array}$ & 3.01 & 1.08 & 3.25 & $++^{* *}$ \\
\hline 40. & $\begin{array}{l}\text { Salvia moorcroftiana Wall. ex } \\
\text { Benth. }\end{array}$ & 73.2 & 2.14 & 156.64 & $++^{* *}$ \\
\hline 41. & Teucrium stocksianum Boiss. & 58.73 & 0.29 & 16.78 & $+*$ \\
\hline 42. & Astragalus pyrrhotrichus Boiss. & 23.61 & 2.28 & 53.83 & $++* *$ \\
\hline 43. & Plantago lanceolata L. & 4.54 & 0.61 & 2.76 & $+*$ \\
\hline 44. & $\begin{array}{l}\text { Limonium macrorhabdon (Boiss.) } \\
\text { O. Kuntze, Rev. Gen. }\end{array}$ & 13.17 & 0.95 & 12.57 & $+*$ \\
\hline 45. & $\begin{array}{l}\text { Polygala abyssinica R.Br. ex } \\
\text { Fresen. }\end{array}$ & 6.29 & 0.88 & 5.51 & $+*$ \\
\hline 46. & Emex spinosa (L.) Campd. & 96.01 & 0.79 & 75.5 & $+*$ \\
\hline 47. & $\begin{array}{c}\text { Persicaria glabra (Willd.) M. } \\
\text { Gómez }\end{array}$ & 56.43 & 0.69 & 38.81 & $+^{*}$ \\
\hline 48. & Cheilanthes pteridoides C. Chr. & 8.77 & 1 & 8.77 & $++* *$ \\
\hline 49. & Pteris cretica L. & 13.95 & 3.16 & 43.47 & $++* *$ \\
\hline 50. & $\begin{array}{l}\text { Delphinium uncinatum Hook.f. \& } \\
\text { Thomson }\end{array}$ & 4.04 & 2.16 & 8.74 & $++* *$ \\
\hline 51. & Delphinium suave Huth & 36.12 & 1.24 & 44.76 & $++* *$ \\
\hline 52. & Ranunculus arvensis L. & 65.97 & 0.5 & 32.76 & $+*$ \\
\hline 53. & Duchesnea indica (Jacks.) Focke & 38.02 & 0.49 & 18.64 & $+*$ \\
\hline 54. & Sanguisorba minor Scop. & 4.39 & 1.55 & 6.79 & $++* *$ \\
\hline 55. & Rosa macrophylla Lindl. & 134.97 & 0.31 & 41.52 & $+^{*}$ \\
\hline 56. & Verbascum thapsus L. & 56.48 & 0.69 & 38.71 & $+*$ \\
\hline 57. & $\begin{array}{l}\text { Wulfeniopsis amherstiana (Wall. } \\
\text { Ex Benth.) D.Y. Hong }\end{array}$ & 124.91 & 1.03 & 129.21 & $++* *$ \\
\hline 58. & Solanum nigrum L., Sp. Pl. & 13.68 & 3.76 & 51.41 & $++* *$ \\
\hline 59. & Daphne mucronata Royle & 8.95 & 1.92 & 17.22 & $++* *$ \\
\hline 60. & Urtica pilulifera $\mathrm{L}$. & 16.09 & 0.13 & 2.11 & $+*$ \\
\hline 61. & Verbena officinalis L. & 9.66 & 4.1 & 39.61 & $++* *$ \\
\hline
\end{tabular}

Bioconcentration Factor $(\mathrm{BCF})=$ Conc. of lead in root $\div$ Conc. of lead in Soil, Translocation Factor $(\mathrm{TF})=$ Conc. of lead in Shoot $\div$ Conc. of lead in root, Bioaccumulation Coefficient $(B A C)=$ Conc. of lead in Shoot $\div$ Conc. of lead in soil, $+^{*}=$ Metal excluders; may be used for the phytostabilization of metal, $++^{* *}=$ Metal indicators; May be used for the phytoextraction of metal, +++*** = Metal hyper accumulator; may be used for the Phytoextraction and recovery of metal., -; cannot be used for the metal remediation processes

\section{Conclusions}

Among the studied sites the concentration of lead in the soil of site 30 was found higher $(32.3 \mathrm{mg} / \mathrm{kg})$ than the allowable limit while in the rest of the sites it is less than the allowable limit. The concentration of lead was found more than the permissible limits in the roots and shoots of all the analyzed plants. The BCFs, TFs and BACs values of the plants for lead metal was found in the range of 1.86-193.33, 0.05-8.3 and 1.79-219 respectively. Among the analysed plants, based on the concentration of lead in shoots and BCFs, TFs and BACs values, Erigeron canadensis L., Stellaria media (L.) Vill. Medicago minima (L.) L.,
Delphinium uncinatum Hook. f. \& Thomson and Verbena officinalis L. were found the most efficient plants for the phytoextraction of lead. Based on lead concentration in roots and BCFs, TFs and BACs values, Nonea edgeworthii A. DC., Arabidopsis thaliana (L.) Heynh. Sarcococca saligna (D. Don) Muell.-Arg. in DC., Prodr., Marrubium vulgare L. and Ranunculus arvensis L. were found best for the phytostabilization of lead metal. Based on the findings of this research work it is recommended that the plants which were found efficient for the phytoremediation; phytoextraction and phytostabilization may be used for the lead remediation purposes 
from the lead contaminated sites. Further research work on the mentioned efficient plants is required to determine the tolerance capacity of these plants to lead contamination in their habitats.

\section{Authors' contributions}

Conceived and designed the experiments: MS Khan \& $\mathrm{H}$ Ali, Performed the experiments: MS Khan, Analyzed the data: MS Khan, Contributed materials/ analysis/ tools: MS Khan, Wrote the paper: MS Khan \& ZU Nisa.

\section{References}

1. Khan S, Hesham AEL, Qiao M, Rehman S \& He JZ (2010). Effects of $\mathrm{Cd}$ and $\mathrm{Pb}$ on soil microbial community structure and activities. Environ Sci Pollut Res 17: 288-296.

2. Cempel M \& Nikel G (2006). Nickel: A Review of Its Sources and Environmental Toxicology. Polish J of Environ Stud 15(3): 375-382.

3. Dabonne, S, Koffi B, Kouadio E, Koffi A, Due E \& Kouame L (2010). Traditional utensils: Potential sources of poisoning by heavy metals. $\mathrm{Br} J$ Phar Toxicol 1: 90-92.

4. Mahaffey KR (1990). Environmental lead toxicity: nutrition as a component of intervention. Environ Health Perspect 89: 75-78.

5. Rubin R \& Strayer D (2008). Environmental and Nutritional pathology. Rubins pathology; Clinicopathologic Foundations of Medicine (5th ed.) Lippincot Williams $\&$ Wilkins.

6. Sokol RZ \& Berman N (1991). The effect of age of exposure on leadinducedtesticular toxicity. Toxicology 69: 269-78.

7. Lasat MM (2002). Phytoextraction of toxic metals: a review of biological mechanisms. J Environ Qual 31(1): 109-120.

8. Ali H, Khan E \& Sajad MA (2013). Phytoremediation of heavy metalsConcepts and applications. Chemosphere 91(7): 869-881.
9. Angle J, Chaney R, Li YM \& Baker A (2001). The risk associated with the introduction of native and hyperaccumulators plants. Abstract. USDA, Agricultural Research Service, USA.

10. Vangronsveld J, Herzig R, Weyens N, Boulet J, Adriaensen K, Ruttens A, Thewys T, Vassilev A, Meers E, Nehnevajova E, Van der Lelie D \& Mench M (2009). Phytoremediation of contaminated soils and groundwater: lessons from the field. Environ Sci Pollut Res 16: 765-794.

11. Adesodun JK, Atayese MO, Agbaje TA, Osadiaye BA, Mafe OF \& Soretire AA (2010). Phytoremediation potentials of sunflowers (Tithonia diversifolia and Helianthus annus) for Metals in soils contaminated with zinc and lead nitrates. Water, Air and Soil Pollut 207(1): 195-201.

12. Padmavathiamma PK \& Li LY (2007). Phytoremediation technology: Hyperaccumulation metals in plants. Water, Air and Soil Pollut 184(1): 105126.

13. Zhuang $\mathrm{P}$, Yang QW, Wang HB \& Shu WS (2007). Phytoextraction of heavy Metals by eight plant species in the field. Water, Air and Soil Pollut 184(1): 235-242.

14. Sharidah MMA (1999). Heavy metals in mangrove sediment of united Arab Emirates Shoreline (Arab Gulf). Water Air Soil Pollut 116(3): 523-534.

15. Awofolu OR (2005). A survey of trace metals in vegetation, soil, and lower animals along some selected major roads in metropolitan city of Lagos. Environ Monit Assess 105(1):431 447.

16. Li MS, Luo YP \& Su ZY (2007). Heavy metal concentrations in soils and plant accumulation in a restored manganese mine land in Guangxi, South China. Environ Pollut 147(1): 168-175.

17. Cui S, Zhou Q \& Chao ZY (2007). Potential hyper-accumulation of $\mathrm{Pb}$, $\mathrm{Zn}, \mathrm{Cu}$ and $\mathrm{Cd}$ in endurant plants 
distributed in an old smeltery, northeast China. Environ Geology 51(6): 1043- 1048.

18. Malik RN, Husain SZ, Nazir I (2010). Heavy Metal Contamination and Accumulation in Soil and Wild Plant Species from Industrial Area of Islamabad, Pakistan. Pak J Bot 42(1): 291-301.

19. Nergus Y (2002). Problems of health and environmental geochemistry. Thesis, Hamdard University, Karachi, Pakistan. 85-104.

20. Nazir R, Khan M, Masab M, Rehman HU, Rauf NU, Shahab S, Ameer N, Sajed M, Ullah M, Rafeeq M \& Shaheen Z (2015). Accumulation of Heavy Metals ( $\mathrm{Ni}, \mathrm{Cu}, \mathrm{Cd}, \mathrm{Cr}, \mathrm{Pb}, \mathrm{Zn}$, $\mathrm{Fe}$ ) in the soil, water and plants and analysis of physico-chemical parameters of soil and water Collected from Tanda Dam kohat. J Pharm Sci \& $\operatorname{Res}$ 7(3): 89-97.

21. Nazir A, Malik RN, Ajaib M, Khan N \& Siddiqui MF (2011).
Hyperaccumulators of heavy metals of industrial areas of Islamabad and Rawalpindi. Pak J Bot 43(4): 19251933.

22. Yoon J, Cao X, Zhou Q \& Ma LQ (2006). Accumulation of $\mathrm{Pb}, \mathrm{Cu}$ and $\mathrm{Zn}$ in native plants growing on contaminated Florida site. Sci of the total Environ 368(2-3): 456-464.

23. Sheoran V, Sheoran A \& Poonia P (2011). Role of hyperaccumulators in phytoextraction of metals from contaminated mining sites: a review. Crit Rev Environ Sci Technol 41(2): 168-214.

24. Fitz WJ \& Wenzel WW (2002). Arsenic transformation in the soilrhizosphere- plant system, fundamentals and potential application of phytoremediation. J Biotechnol 99: 259-278.

25. Jamil S, Abhilash PC, Singh N \& Sharma PN (2009). A potential crop for phytoremediation of coal fly ash. $J$ of Hazard Mater 172(1): 269-275. 